\title{
FUNDAMENTAL SOLUTIONS FOR DIRAC-TYPE OPERATORS
}

\author{
SWANHILD BERNSTEIN \\ Freiberg University of Mining and Technology \\ Faculty of Mathematics and Computer Science \\ Institute of Applied Mathematics I \\ D-09596 Freiberg, Germany
}

\begin{abstract}
We consider the Dirac-type operators $D+a, a$ is a paravector in the Clifford algebra. For this operator we state a Cauchy-Green formula in the spaces $C^{1}(G)$ and $W_{p}^{1}(G)$. Further, we consider the Cauchy problem for this operator.

1. Preliminaries. Dirac and Dirac-type operators are considered in many papers. A significant selection of papers is contained in the bibliography. Most of them consider the operators in the quaternionic algebra. We want to consider the operator in the Clifford algebra. Thus our considerations differ in some sense from the considerations in the quaternionic algebra. We consider stationary problems and obtain as the main result the Cauchy-Green formula. In the case of nonstationary problems we consider the Cauchy problem.
\end{abstract}

2. Introduction. Let $\left(e_{1}, \ldots, e_{m}\right)$ be an orthonormal basis of $\mathbb{R}^{m}, m \in \mathbb{N}$, then by $\mathcal{C}$ we denote the $2^{m}$-dimensional Clifford algebra obtained from the generating relations $e_{j} e_{k}+e_{k} e_{j}=2 \delta_{j k}, j, k=1, \ldots, m$. Thus the quaternionic case is not contained. An element of $\mathcal{C}$ is of the form $a=\sum a_{A} e_{A}, a_{A} \in \mathbb{C}, e_{A}=e_{a_{1} \ldots a_{k}}=e_{a_{1}} \cdot \ldots \cdot e_{a_{k}}$ for $A=\left\{a_{1}, \ldots, a_{k}\right\}$ with $a_{1}<\ldots<a_{k}, e_{0}=1$ is the identity of $\mathcal{C}$. We identify a vector $x \in \mathbb{R}^{m}$ with the element $x=\sum_{j=1}^{m} x_{j} e_{j}$ of the Clifford algebra. Let $G$ be a bounded domain of $\mathbb{R}^{m}$, with smooth boundary $\Gamma$. If $F$ is a functionspace of complex-valued function, a function $u=\sum u_{A} e_{A}$ is an element of $F_{\mathcal{C}}$ iff $u_{A}$ is a complex-valued function of the space $F$. We use the Sobolev-spaces $W_{p}^{k}(G), 1<p<\infty, k \in \mathbb{N}$, the space of continuously differentiable functions $C^{1}(G)$ and the space $L_{2}\left[0, t ; L_{2}\left(\mathbb{R}^{m}\right)\right]$ with the norm

1991 Mathematics Subject Classification: Primary 30G35, Secondary 47G10.

The paper is in final form and no version of it will be published elsewhere. 
$\left(\int_{0}^{T}\|f\|_{L_{2}\left(\mathbb{R}^{m}\right)}^{2} d t\right)^{1 / 2}$. We consider the Dirac operator

$$
D_{x}+a=\sum_{j=1}^{m} e_{j}\left(\frac{\partial}{\partial x_{j}}+a_{j}\right)+e_{0} a_{0}, \quad m \in \mathbb{N},
$$

where $a=\sum_{j=1}^{m} e_{j} a_{j}=a_{0} e_{0}+\underline{a}$ and $D_{x}=\sum_{j=1}^{m} e_{j} \frac{\partial}{\partial x_{j}}$, then $D_{x}^{2}=\nabla, \nabla$ being the Laplacian. We want to illustrate the Dirac-operator in the Pauli-algebra. The basic vectors are $e_{1}, e_{2}, e_{3}, e_{1}^{2}=e_{2}^{2}=e_{3}^{2}=1$, the unit element of the algebra is $e_{0}$. We have the bivectors $e_{23}=e_{2} e_{3}, e_{31}=e_{3} e_{1}$ and $e_{12}=e_{1} e_{2}$ and the pseudoscalar $e_{123}=e_{1} e_{2} e_{3}$. We consider a function

$$
u=u_{0} e_{0}+u_{1} e_{1}+u_{2} e_{2}+u_{3} e_{3}+u_{23} e_{23}+u_{31} e_{31}+u_{12} e_{12}+u_{123} e_{123} .
$$

The scalar part is $u_{0}$, the pseudoscalar part $u_{123}$, the vector $\underline{u}=\left(u_{1}, u_{2}, u_{3}\right)$ and the bivector $\underline{v}=\left(u_{23}, u_{31}, u_{12}\right)$. Then we obtain for the Dirac operator

$$
\begin{gathered}
D u=\sum_{i=1}^{3} e_{i} \frac{\partial}{\partial x_{i}} u=+\left(\frac{\partial}{\partial x_{1}} u_{1}+\frac{\partial}{\partial x_{2}} u_{2}+\frac{\partial}{\partial x_{3}} u_{3}\right) e_{0}+ \\
+\left(\frac{\partial}{\partial x_{3}} u_{31}-\frac{\partial}{\partial x_{2}} u_{12}+\frac{\partial}{\partial x_{1}} u_{0}\right) e_{1}+\left(\frac{\partial}{\partial x_{1}} u_{12}-\frac{\partial}{\partial x_{3}} u_{23}+\frac{\partial}{\partial x_{2}} u_{0}\right) e_{2}+ \\
+\left(\frac{\partial}{\partial x_{2}} u_{31}-\frac{\partial}{\partial x_{1}} u_{31}+\frac{\partial}{\partial x_{3}} u_{0}\right) e_{3}+ \\
+\left(\frac{\partial}{\partial x_{2}} u_{3}-\frac{\partial}{\partial x_{3}} u_{2}+\frac{\partial}{\partial x_{1}} u_{123}\right) e_{23}+\left(\frac{\partial}{\partial x_{3}} u_{1}-\frac{\partial}{\partial x_{1}} u_{3}+\frac{\partial}{\partial x_{2}} u_{123}\right) e_{31}+ \\
+\left(\frac{\partial}{\partial x_{1}} u_{2}-\frac{\partial}{\partial x_{2}} u_{1}+\frac{\partial}{\partial x_{3}} u_{123}\right) e_{12}+\left(\frac{\partial}{\partial x_{3}} u_{23}-\frac{\partial}{\partial x_{2}} u_{31}+\frac{\partial}{\partial x_{3}} u_{12}\right) e_{123} .
\end{gathered}
$$

This system is equivalent to another system

$$
\begin{gathered}
\operatorname{div} \underline{u}=-* \operatorname{curl} * \underline{v} \\
+\operatorname{curl} * \underline{u}-* \operatorname{grad} * u_{123} \\
-* \operatorname{div} * \underline{v},
\end{gathered}
$$

where $*$ is the Hodge-operator (multiplication with the pseudo scalar $e_{123}$ ),

$$
\begin{gathered}
* e_{0}=e_{123}, \quad * e_{123}=-e_{0} ; \quad * e_{2}=e_{31}, \quad * e_{31}=-e_{2} ; \\
* e_{1}=e_{23}, \quad * e_{23}=-e_{1} ; \quad * e_{3}=e_{12}, \quad * e_{12}=-e_{3} ;
\end{gathered}
$$

and the operator div is defined for vector $\underline{u}$

$$
\operatorname{div} \underline{u}=\sum_{i=1}^{3} \frac{\partial}{\partial x_{i}} u_{i},
$$

and the operator curl is also defined for vectors $\underline{u}$ in the following way

$$
\operatorname{curl} \underline{u}=\left(\frac{\partial}{\partial x_{2}} u_{3}-\frac{\partial}{\partial x_{3}} u_{2}\right) e_{23}+\left(\frac{\partial}{\partial x_{3}} u_{1}-\frac{\partial}{\partial x_{1}} u_{3}\right) e_{31}+\left(\frac{\partial}{\partial x_{1}} u_{2}-\frac{\partial}{\partial x_{2}} u_{1}\right) e_{12}
$$

and the Hodge-operator transforms bivectors in vectors and vectors in bivectors. The 
operator grad is defined for scalars $u_{0}$

$$
\operatorname{grad} u_{0}=\sum_{i=1}^{3} \frac{\partial}{\partial x_{i}} u_{0} e_{i}
$$

and the Hodge-operator transforms scalars in pseudoscalars and pseudoscalars in scalars.

3. The Dirac-type operator $D+a$. Let $m$ be a natural number and

$$
K_{a_{0}}(x)=K_{a_{0}}(|x|)=\frac{1}{\pi^{2 / m} 2^{m / 2}}\left({\frac{a_{0}}{|x|}}^{m / 2-1} K_{\frac{m}{2}-1}\left(a_{0}|x|\right)\right)
$$

where $K$ denotes modified Bessel functions, the so-called MacDonald functions.

LEMma. The fundamental solution for $\nabla-a_{0}^{2}$ is $K_{a_{0}}$.

The proof is contained in [0rt].

THEOREM. The fundamental solution for $D_{x}+a$ is

$$
\begin{gathered}
E_{a}(x)=\exp ^{-<\underline{a}, x>}\left\{\left(D_{x}-a_{0}\right) K_{a_{0}}(x)\right\}= \\
=\exp ^{-<\underline{a}, x>}\left\{\frac{1}{a_{0}(2 \pi)^{m / 2}} \cdot \sum_{j=1}^{m} \frac{x_{j} e_{j}}{|x|^{m}}\left(a_{0}|x|\right)^{m / 2} K_{m / 2}\left(a_{0}|x|\right)+\right. \\
\left.+\frac{a_{0}}{(2 \pi)^{m / 2}} \cdot \frac{1}{|x|^{m-2}}\left(a_{0}|x|\right)^{m / 2-1} K_{m / 2-1}\left(a_{0}|x|\right)\right\}, \quad \text { with }\langle\underline{a}, x\rangle=\sum_{i=1}^{m} a_{i} x_{i} .
\end{gathered}
$$

Proof. We prove that $E_{a}(x)$ is locally integrable and $(D+a) E_{a}=0$ in $\mathbb{R}^{m} \backslash\{0\}$ and $(D+a) E_{a}=\delta$. On every compact subset of $\mathbb{R}^{m}$ the function $e^{-<\underline{a}, x>}$ is bounded from above and positive,

$$
\begin{aligned}
K_{a_{0}} & \sim \frac{1}{|x|^{m-2}}, \quad|x| \rightarrow 0, \quad K_{a_{0}} \sim \frac{e^{-\left|\mathcal{R} a_{0}\right| \cdot|x|}}{|x|^{m / 2-1 / 2}}, \quad|x| \rightarrow \infty, \\
\frac{\partial K_{a_{0}}(x)}{\partial x_{j}} & \sim \frac{x_{j}}{|x|^{m}}, \quad|x| \rightarrow 0, \quad \frac{\partial K_{a_{0}}(x)}{\partial x_{j}} \sim \frac{e^{-\left|\mathcal{R} a_{0}\right| \cdot|x|} x_{j}}{|x|^{m / 2+1}}, \quad|x| \rightarrow \infty,
\end{aligned}
$$

$\mathcal{R} a_{0}$ denotes the real part of the complex number $a_{0}$. Thus $E_{a}(x)$ is locally integrable in $\mathbb{R}^{m}$. Next,

$$
\begin{gathered}
\left(D_{x}+a\right) E_{a}(x)=\left(D_{x}+a\right)\left\{e^{-<\underline{a}, x>}\left(D_{a}-a_{0}\right) K_{a_{0}}(x)\right\}= \\
=-\underline{a} e^{-<\underline{a}, x>}\left(D_{x}-a_{0}\right) K_{a_{0}}(x)+e^{-<\underline{a}, x>} D_{x}\left(D_{x}-a_{0}\right) K_{a_{0}}(x)+ \\
+a e^{-<\underline{a}, x>}\left(D_{x}-a_{0}\right) K_{a_{0}}(x)= \\
=e^{-<\underline{a}, x>}\left(D_{x}+a_{0}\right)\left(D_{x}-a_{0}\right) K_{a_{0}}(x)=e^{-<\underline{a}, x>}\left(\Delta-a_{0}^{2}\right) K_{a_{0}}(x)=0
\end{gathered}
$$

in $\mathbb{R}^{m} \backslash\{0\}$. On the other hand, let $\phi \in \mathcal{D}\left(\mathbb{R}^{m}\right)$ :

$$
\begin{gathered}
\left((D+a) E_{a}(x), \phi(x)\right)=\left(e^{-<\underline{a}, x>}\left(\Delta-a_{0}^{2}\right) K_{a_{0}}(x), \phi(x)\right)= \\
=\left(\left(\Delta-a_{0}^{2}\right) K_{a_{0}}(x), e^{-<\underline{a}, x>} \phi(x)\right)=\left(\delta, e^{-<\underline{a}, x>} \phi(x)\right)= \\
=\phi(0)=(\delta, \phi) .
\end{gathered}
$$

R e mark. The case $a=a_{0} \in \mathbb{C}$ is discussed in $[\mathrm{Xu}]$ if $e_{i}^{2}=-1$ by using outer and inner monogenics. 
We introduce the integral operators

$$
T_{a} u:=\int_{G} E_{a}(x-y) u(y) d y, \quad F_{a} u:=-\int_{\Gamma} E_{a}(x-y) n(y) u(y) d y, \quad x \notin \Gamma .
$$

Here, $n(y)$ denotes the outward-normal at the point $y \in \Gamma$.

THEOREM. The operator

$$
T_{a}: W_{p, c}^{k}(G) \rightarrow W_{p, C}^{k+1}(g), \quad 1<p<\infty, k=0,1, \ldots
$$

is continuous.

Proof. $T_{a}$ is a weakly singular integral operator with

$$
\left|E_{a}(x-y)\right| \leq \frac{C}{|x-y|^{m-1}}
$$

and using $[\mathrm{MP}]$ we get thus the operator

$$
T_{a}: L_{p, C}(G) \rightarrow L_{p, C}(g), \quad 1<p<\infty
$$

is continuous. The rest is contained in the following lemmas.

Lemma. Let $u \in L_{p, C}(G), 1<p<\infty, k=0,1, \ldots$ Then

$$
\frac{\partial}{\partial x_{k}} T_{a} u=\int_{G} \frac{\partial}{\partial x_{k}} E_{a}(x-y) u(y) d y+\frac{u(x)}{A_{m}} \int_{S_{1}} \frac{y-x}{|x-y|} \cdot \frac{y_{k}-x_{k}}{|x-y|} d S_{1}(y),
$$

where the integral over $S_{1}$ is a constant only depending on the dimension $m$ and $k$ and $A_{m}=\frac{2 \pi^{m / 2}}{\Gamma\left(\frac{m}{2}\right)}$ is the area of the unit sphere in $\mathbb{R}^{m}$.

Proof. We have $E_{a}(x-y)=e^{-<\underline{a}, x-y>}\left(D_{x}-a_{0}\right) K_{a_{0}}(x-y)$,

$$
\begin{gathered}
a_{0} K_{a_{0}}(x-y) \sim \frac{1}{|x-y|^{m-2}}, \quad x \rightarrow y, \\
D_{x} K_{a_{0}}(x-y)=\frac{1}{A_{m}} e^{-<\underline{a}, x-y>} \frac{x-y}{|x-y|}, \quad x \rightarrow y .
\end{gathered}
$$

Now, we get

$$
\begin{gathered}
\quad \frac{\partial}{\partial x_{k}} \int_{G_{\epsilon}} e^{-<\underline{a}, x-y>}\left(D_{x}-a_{0}\right) K_{a_{0}}(x-y) u(y) d y= \\
\int_{G_{\epsilon}} \frac{\partial}{\partial x_{k}}\left\{e^{-<\underline{a}, x-y>}\left(D_{x}-a_{0}\right) K_{a_{0}}(x-y)\right\} u(y) d y+ \\
-\int_{r=\epsilon=|x-y|} e^{-<\underline{a}, x-y>}\left(D_{x}-a_{0}\right) K_{a_{0}}(x-y) u(y) \cos \left(r, x_{k}\right) d S_{\epsilon} \\
=\int_{G_{\epsilon}} \frac{\partial}{\partial x_{k}}\left\{e^{-<\underline{a}, x-y>}\left(D_{x}-a_{0}\right) K_{a_{0}}(x-y)\right\} u(y) d y+ \\
+\int_{\epsilon=|x-y|} e^{-<\underline{a}, x-y>} a_{0} K_{a_{0}}(x-y) u(y) \cos \left(r, x_{k}\right) d S_{\epsilon}+ \\
-\int_{\epsilon=|x-y|} e^{-<\underline{a}, x-y>} D_{x} K_{a_{0}}(x-y) u(y) \cos \left(r, x_{k}\right) d S_{\epsilon}= \\
=\int_{G_{\epsilon}} \frac{\partial}{\partial x_{k}}\left\{e^{-<\underline{a}, x-y>}\left(D_{x}-a_{0}\right) K_{a_{0}}(x-y)\right\} u(y) d y+
\end{gathered}
$$




$$
\begin{aligned}
& -\int_{|x-y|=1} \epsilon^{m-1} e^{-<\underline{a}, x-(x+\epsilon \theta)>} a_{0} K_{a_{0}}(x-y) u(x+\epsilon \theta) \cos \left(r, x_{k}\right) d S_{1}+ \\
& -\int_{|x-y|=1} e^{-<\underline{a}, x-(x+\epsilon \theta)>} D_{x} K_{a_{0}}(x-y) u(x+\epsilon \theta) \epsilon^{m-1} \cos \left(r, x_{k}\right) d S_{1} .
\end{aligned}
$$

We take $\epsilon \rightarrow 0$ :

$$
\begin{gathered}
\int_{G} \frac{\partial}{\partial x_{k}} E_{a}(x-y) u(y) d y+\frac{u(x)}{A_{m}} \int_{S_{1}} \frac{y-x}{|x-y|} \cdot \frac{y_{k}-x_{k}}{|x-y|} d S_{1}(x), \\
\left.e^{-<\underline{a}, x-(x+\epsilon \theta)>}=e^{\epsilon<\underline{a}, \theta>} \rightarrow 1\right) .
\end{gathered}
$$

Lemma. The operator $\frac{\partial}{\partial x_{k}} T_{a}: L_{p, C}(g) \rightarrow L_{p, C}, 1<p<\infty$, is continuous.

Proof. From the Lemma above we get

$$
\frac{\partial}{\partial x_{k}} T_{a}=\int_{G} \frac{\partial}{\partial x_{k}} E_{a}(x-y) u(y) d y+C(k) u(x),
$$

where $C(k)$ is a constant only depending on $k$. Thus $C(k) u \in L_{p, C}$ if $u \in L_{p, C}$. We consider the first term.

$$
\begin{gathered}
\frac{\partial}{\partial x_{k}} E_{a}(x-y)= \\
-\underline{a} E_{a}(x-y)-e^{-<\underline{a}, x-y>} \frac{a_{0}}{(2 \pi)^{m / 2}} \cdot \frac{x_{k}-y_{k}}{|x-y|^{m}}\left(a_{0}|x-y|\right)^{m / 2} K_{m / 2}\left(a_{0}|x-y|\right)+ \\
e^{-<\underline{a}, x-y>} \frac{a_{0}}{(2 \pi)^{m / 2}} \cdot \sum_{j=1}^{m} \frac{\left(x_{j}-y_{j}\right)\left(x_{k}-y_{k}\right)}{|x-y|^{m}} \cdot\left(a_{0}|x-y|\right)^{m / 2-1} K_{m / 2-1}\left(a_{0}|x-y|\right)+ \\
e^{-<\underline{a}, x-y>} \frac{1}{a_{0}(2 \pi)^{m / 2}} K_{m / 2-}\left(a_{0}|x-y|\right) \cdot \sum_{j=1}^{m} \frac{\partial}{\partial x_{k}}\left(\frac{\left(x_{j}-y_{j}\right) e_{j}}{|x-y|}\right) .
\end{gathered}
$$

We only have to consider the last part, because the other parts lead to weakly singular kernels. We prove that the last part creates a singular kernel of a Calderon-Zygmund operator. We choose $k=1$ and use sphericals cordinates in the following way:

$$
\begin{gathered}
y_{1}=x_{1}+r \cos \theta_{1}, \\
y_{2}=x_{2}+r \sin \theta_{1} \cos \theta_{1}, \\
\ldots \ldots ., \\
y_{m-1}=x_{m-1}+r \sin \theta_{1} \sin \theta_{2} \ldots \sin \theta_{m-2} \cos \theta_{m-1}, \\
y_{m}=x_{m}+r \sin \theta_{1} \sin \theta_{2} \ldots \sin \theta_{m-2} \sin \theta_{m-1},
\end{gathered}
$$

$r=|x-y|, \theta_{1} \in[0, \pi], \theta_{i} \in[-\pi, \pi], i=2,3, \ldots, m-1$. We have

$$
\frac{\partial r}{\partial x_{1}}=\frac{x_{1}-y_{1}}{r}=-\cos \theta_{1} \text {. }
$$

Thus

$$
-\frac{\partial}{\partial x_{1}} \cos \theta_{1}=\frac{1}{r}-\frac{\left(x_{1}-y_{1}\right)^{2}}{r^{3}} \frac{1}{r}-\frac{\cos ^{2} \theta_{1}}{r}=\frac{\sin ^{2} \theta_{1}}{r}=\sin \theta_{1} \frac{\partial \theta_{1}}{\partial x_{1}}
$$

and we obtain

$$
\frac{\partial \theta_{1}}{\partial x_{1}}=\frac{\sin \theta_{1}}{r}
$$


because of

$$
\theta_{1} \in[0, \pi]
$$

Further

$$
\begin{gathered}
\frac{\partial}{\partial x_{1}}\left(\frac{y_{2}-x_{2}}{r}\right)=\frac{\partial}{\partial x_{1}}\left(\sin \theta_{1} \cos \theta_{2}\right) \\
=\frac{\partial}{\partial \theta_{1}}\left(\sin \theta_{1} \cos \theta_{2}\right) \frac{\partial \theta_{2}}{\partial x_{1}}=\frac{\sin \theta_{1}}{r} \cos \theta_{1} \cos \theta_{2}-\sin \theta_{1} \sin \theta_{2} \frac{\partial \theta_{2}}{\partial x_{1}} \\
\cos \theta_{1} \cos \theta_{2} \frac{\partial \theta_{1}}{\partial x_{1}}-\sin \theta_{1} \sin \theta_{2} \frac{\partial \theta_{2}}{\partial x_{1}}=\frac{\sin \theta_{1}}{r} \cos \theta_{1} \cos \theta_{2}-\sin \theta_{1} \sin \theta_{2} \frac{\partial \theta_{2}}{\partial x_{1}} .
\end{gathered}
$$

On the other hand

$$
\frac{\partial}{\partial x_{1}}\left(\frac{y_{2}-x_{2}}{r}\right)=\frac{\left(y_{2}-x_{2}\right)}{r} \cdot\left(\frac{\left(y_{1}-x_{1}\right)}{r}\right) \cdot \frac{1}{r}=\frac{\sin \theta_{1}}{r} \cos \theta_{1} \cos \theta_{2}
$$

and we obtain

$$
-\sin \theta_{1} \sin \theta_{2} \frac{\partial \theta_{2}}{\partial x_{1}}=0
$$

and thus $\frac{\partial \theta_{2}}{\partial x_{1}}=0$. Now, let $\frac{\partial \theta_{j}}{\partial x_{1}}=0, j=2, \cdot l-1, l \leq m-1$, then

$$
\begin{gathered}
\frac{\partial}{\partial x_{1}}\left(\frac{y_{2}-x_{2}}{r}\right)=\frac{\partial}{\partial x_{1}}\left(\sin \theta_{1} \cdot \ldots \cdot \sin \theta_{l-1} \cos \theta_{l}\right) \\
=\frac{\partial}{\partial \theta_{1}}\left(\sin \theta_{1} \cdot \ldots \cdot \sin \theta_{l-1} \cos \theta_{l}\right) \frac{\partial \theta_{1}}{\partial x_{1}}+\frac{\partial}{\partial \theta_{1}}\left(\sin \theta_{1} \ldots \cdot \sin \theta_{l-1} \cos \theta_{l}\right) \frac{\partial \theta_{l}}{\partial x_{1}}= \\
\frac{\sin \theta_{1}}{r} \cos \theta_{1} \sin \theta_{2} \ldots \sin \theta_{l-1} \cos \theta_{l}-\sin \theta_{1} \ldots \sin \theta_{l-1} \sin \theta_{1} \frac{\partial \theta_{1}}{\partial x_{1}} .
\end{gathered}
$$

On the other hand

$$
\frac{\partial}{\partial x_{1}}\left(\frac{y_{1}-x_{1}}{r}\right)=\frac{\left(y_{1}-x_{1}\right)}{r} \cdot\left(\frac{\left(y_{1}-x_{1}\right)}{r}\right) \cdot \frac{1}{r}=\frac{\sin \theta_{1}}{r} \cos \theta_{1} \sin \theta_{2} \ldots \sin \theta_{l-1} \cos \theta_{l}
$$

and we get $\frac{\partial \theta_{l}}{\partial x_{1}}=0, l=2, \ldots, m-1$. Though, we obtain

$$
\begin{aligned}
& \frac{\partial}{\partial x_{1}}\left(\frac{x}{|x|^{m}}\right)=\frac{\partial}{\partial x_{1}}\left(\sum_{j=1}^{m} \frac{\left(x_{j}-y_{j}\right)}{|x-y|^{m}} e_{j}\right)=-\frac{\partial}{\partial x_{1}}\left(\sum_{j=1}^{m} \frac{\left(x_{j}-y_{j}\right)}{r} \cdot \frac{1}{r^{m}} e_{j}\right)= \\
& =\frac{(m-1)}{r^{m}} \cdot \frac{\partial r}{\partial x_{1}} \cdot \sum_{j=1}^{m} \frac{\left(x_{j}-y_{j}\right)}{|x-y|^{m}} e_{j}-\frac{1}{r^{m-1}} \frac{\partial}{\partial \theta_{1}}\left(\sum_{j=1}^{m} \frac{\left(x_{j}-y_{j}\right)}{|x-y|^{m}} e_{j}\right) \frac{\partial \theta_{1}}{\partial x_{1}}= \\
& =-\frac{1}{r^{m}}\left\{r \frac{\partial}{\partial \theta_{1}}\left(\sum_{j=1}^{m} \frac{\left(x_{j}-y_{j}\right)}{|x-y|^{m}} e_{j}\right) \frac{\partial}{\partial x_{1}}-(m-1)\left(\sum_{j=1}^{m} \frac{\left(x_{j}-y_{j}\right)}{|x-y|^{m}} e_{j}\right) \frac{\partial r}{\partial x_{1}}\right\} .
\end{aligned}
$$

Let

$$
\phi\left(\theta_{1}, \theta_{2}, \ldots, \theta_{m-1}\right):=-\sum_{j=1}^{m} \frac{\left(x_{j}-y_{j}\right)}{r} e_{j}
$$

then

$$
\frac{\partial}{\partial x_{1}}\left(\frac{x}{|x|^{m}}\right)=\frac{1}{r^{m}}\left\{r \frac{\partial \phi}{\partial \theta_{1}} \frac{\partial \theta_{1}}{\partial x_{1}}-(m-1) \phi \frac{\partial r}{\partial x_{1}}\right\}=\frac{f(\phi, r)}{r^{m}} .
$$


We get

$$
\begin{gathered}
\frac{\partial}{\partial x_{1}} E_{a}(x-y)= \\
-\underline{a} E_{a}(x-y)-e^{-<\underline{a}, x-y>} \frac{a_{0}}{(2 \pi)^{m / 2}} \cdot \frac{x_{k}-y_{k}}{|x-y|^{m}}\left(a_{0}|x-y|\right)^{m / 2} K_{m / 2}\left(a_{0}|x-y|\right)+ \\
e^{-<\underline{a}, x-y>} \frac{a_{0}}{(2 \pi)^{m / 2}} \cdot \sum_{j=1}^{m} \frac{\left(x_{j}-y_{j}\right)\left(x_{k}-y_{k}\right)}{|x-y|^{m}} \cdot\left(a_{0}|x-y|\right)^{m / 2-1} K_{m / 2-1}\left(a_{0}|x-y|\right)+ \\
e^{-<\underline{a}, x-y>} \frac{1}{a_{0}(2 \pi)^{m / 2}} \cdot\left(a_{0}|x-y|\right)^{m / 2} K_{m / 2}\left(a_{0}|x-y|\right) \cdot \frac{f(\phi,|x-y|)}{|x-y|^{m}} .
\end{gathered}
$$

We have to prove that $\int_{S_{1}} f(\phi, 1) d S_{1}=0$, where $S_{1}$ is the unit sphere in $\mathbb{R}^{m}$ with center in $x$, and that $\int_{S_{1}}|f(\phi, 1)|^{p^{\prime}} d S_{1}=$ const, $1<p^{\prime}<\infty$. Because of

$$
d S_{1}=\sin ^{m-2} \theta_{1} \sin ^{m-3} \theta_{2} \ldots \sin \theta_{m-2} d \theta_{1} d \theta_{2} \ldots d \theta_{m-2} d \theta_{m-1},
$$

we obtain

$$
\begin{gathered}
\int_{S_{1}} f(\phi, 1) d S_{1}=\int_{-\pi}^{\pi} d \theta_{m-1} \int_{0}^{\pi} \sin \theta_{m-2} d \theta_{m-2} \cdots \int_{0}^{\pi} \sin ^{m-3} \theta_{2} d \theta_{2} \\
\int_{0}^{\pi} f(\phi, 1) \sin ^{m-2} \theta_{1} d \theta_{1} .
\end{gathered}
$$

We have

$$
f(\phi, 1)=C\left(a_{0}\right)\left\{\frac{\partial \phi}{\partial \theta_{1}} \sin \theta_{1}+(m-1) \phi \cos \theta_{1}\right\}
$$

where $C\left(a_{0}\right)$ is a constant only depending on $a_{0}$. Thus the inner integral is equal to

$$
\begin{gathered}
C\left(a_{0}\right) \int_{0}^{\pi}\left[(m-1) \phi \cos \theta_{1}+\frac{\partial \phi}{\partial \theta_{1}} \sin \theta_{1} \sin ^{m-2} \theta_{1} d \theta_{1}=\right. \\
C\left(a_{0}\right) \int_{0}^{\pi} \frac{\partial}{\partial \theta_{1}}\left[\sin ^{m-1} \theta_{1} \cdot \phi\left(\theta_{1}, \ldots, \theta_{m-1}\right)\right] d \theta_{1}=0
\end{gathered}
$$

and thus

$$
\int_{S_{1}} f(\phi, 1) d S_{1}=0
$$

Furthermore, we have

$$
\begin{gathered}
\frac{\partial \phi}{\partial \theta_{1}} \sin \theta_{1}=-\sin ^{2} \theta_{1} e_{1}+\sin \theta_{1} \cos \theta_{1} \cos \theta_{2} e_{2}+\ldots+ \\
\quad+\sin \theta_{1} \cos \theta_{1} \sin \theta_{2} \ldots \sin \theta_{m-2} \cos \theta_{m-1} e_{m-1}+ \\
\quad+\sin \theta_{1} \cos \theta_{1} \sin \theta_{2} \ldots \sin \theta_{m-2} \sin \theta_{m-1} e_{m}
\end{gathered}
$$

and

$$
\begin{gathered}
\phi \cos \theta_{1}=\cos ^{2} \theta_{1} e_{1}+\sin \theta_{1} \cos \theta_{1} \cos \theta_{2} e_{2}+\ldots+ \\
+\sin \theta_{1} \cos \theta_{1} \sin \theta_{2} \ldots \sin \theta_{m-2} \cos \theta_{m-1} e_{m-1}+ \\
+\sin \theta_{1} \cos \theta_{1} \sin \theta_{2} \ldots \sin \theta_{m-2} \sin \theta_{m-1} e_{m}
\end{gathered}
$$


and thus

$$
\begin{gathered}
\frac{\partial \phi}{\partial \theta_{1}} \sin \theta_{1}+(m-1) \phi \cos \theta_{1}=-1 \cdot e_{1}+m \cdot \cos ^{2} \theta_{1} e_{1}+ \\
+m \cdot \sin \theta_{1} \cos \theta_{1} \cos \theta_{2} e_{2}+\ldots+m \cdot \sin \theta_{1} \cos \theta_{1} \sin \theta_{2} \ldots \sin \theta_{m-2} \sin \theta_{m-1} e_{m}
\end{gathered}
$$

and

and we obtain finally

$$
\left|\frac{\partial \phi}{\partial \theta_{1}} \sin \theta_{1}+(m-1) \phi \cos \theta_{1}\right| \leq 1+m^{2}
$$

$$
\int_{S_{1}}|f(\phi, 1)|^{p^{\prime}} d S_{1} \leq\left(1+m^{2}\right)^{p^{\prime}} \cdot A_{m}, \quad 1<p^{\prime}<\infty .
$$

If $k \neq 1$, we choose spherical coordinates such that the same situation arises.We obtain that $\int_{G} \frac{\partial}{\partial x_{k}} E_{a}(x-y) u(y) d y$ consists of weakly singular integral operators and a CalderonZygmund operator (singular integral operator). This completes the proof.

Lemma. Let $u \in C_{C}^{d}(G)$ then we have

$$
\left(D_{x}+a\right) T_{a} u= \begin{cases}u(x) & \text { in } G \\ 0 & \text { in } \mathbb{R}^{m} \backslash \bar{G} .\end{cases}
$$

This follows immediately from the construction of the operator $T_{a}$.

An important connection between the operators $D_{x}+a, T_{a}$ and $F_{a}$ is given by the Cauchy-Green formula.

Theorem (Cauchy-Green formula). Let $u \in C_{C}^{1}(G)$ then we have

$$
F_{a} u+T_{a}\left(D_{x}+a\right) u= \begin{cases}u(x) & \text { in } G \\ 0 & \text { in } \mathbb{R}^{m} \backslash \bar{G} .\end{cases}
$$

Pr o of. We have

$$
\begin{gathered}
\left(D_{y}-a\right)\left\{e^{-<\underline{a}, x-y>} K_{a_{0}}(x-y) u(y)\right\}+e^{-<\underline{a}, x-y>} K_{a_{0}}(x-y) a u(y)= \\
=\underline{a} e^{-<\underline{a}, x-y>} K_{a_{0}}(x-y) u(y)-e^{-<\underline{a}, x-y>} D_{x} K_{a_{0}}(x-y) u(y)+ \\
+e^{-<\underline{a}, x-y>} K_{a_{0}}(x-y) D_{y} u(y)-a e^{-<\underline{a}, x-y>} K_{a_{0}}(x-y) u(y)+ \\
+e^{-<\underline{a}, x-y>} K_{a_{0}}(x-y) a u(y)= \\
-e^{-<\underline{a}, x-y>}\left(D_{x}+a_{0}\right) K_{a_{0}}(x-y) u(y)+e^{-<\underline{a}, x-y>} K_{a_{0}}(x-y)\left(D_{y}+a_{0}\right) u(y) .
\end{gathered}
$$

Let $G_{\epsilon}=\{y \in G:|x-y|>\epsilon\}$ then

$$
\begin{gathered}
\int_{G_{\epsilon}}\left(D_{y}-a\right)\left\{e^{-<\underline{a}, x-y>} K_{a_{0}}(x-y) u(y)\right\} d y= \\
-\int_{G_{\epsilon}} e^{-<\underline{a}, x-y>}\left(D_{x}+a_{0}\right) K_{a_{0}}(x-y) u(y) d y+ \\
+\int_{G_{\epsilon}} e^{-<\underline{a}, x-y>} K_{a_{0}}(x-y)\left(D_{y}+a\right) u(y) d y a \int_{G_{\epsilon}} e^{-<\underline{a}, x-y>} K_{a_{0}}(x-y) u(y) d y= \\
-\int_{G_{\epsilon}} e^{-<\underline{a}, x-y>}\left(D_{x}+a_{0}\right) K_{a_{0}}(x-y) u(y) d y+\int_{\Gamma} e^{-<\underline{a}, x-y>} K_{a_{0}}(x-y) n(y) u(y) d y+ \\
-\int_{S_{\epsilon}} e^{-<\underline{a}, x-y>} K_{a_{0}}(x-y) n(y) u(y) d y-\underline{a} \int_{G_{\epsilon}} e^{-<\underline{a}, x-y>} K_{a_{0}}(x-y) u(y) d y+
\end{gathered}
$$




$$
\begin{aligned}
& +\int_{G_{\epsilon}} e^{-<\underline{a}, x-y>} D_{x} K_{a_{0}}(x-y) u(y) d y=a \int_{G_{\epsilon}} e^{-<\underline{a}, x-y>} K_{a_{0}}(x-y) u(y) d y+ \\
& +\int_{\Gamma} e^{-<\underline{a}, x-y>} K_{a_{0}}(x-y) n(y) u(y) d y-\int_{S_{\epsilon}} e^{-<\underline{a}, x-y>} K_{a_{0}}(x-y) n(y) u(y) d y .
\end{aligned}
$$

Thus

$$
\begin{gathered}
\int_{G_{\epsilon}} e^{-<\underline{a}, x-y>} K_{a_{0}}(x-y)\left(D_{y}+a\right) u(y) d y-\int_{G_{\epsilon}} e^{-<\underline{a}, x-y>}\left(D_{x}+a_{0}\right) K_{a_{0}}(x-y) u(y) d y= \\
=\int_{\Gamma} e^{-<\underline{a}, x-y>} K_{a_{0}}(x-y) n(y) u(y) d y-\int_{S_{\epsilon}} e^{-<\underline{a}, x-y>} K_{a_{0}}(x-y) n(y) u(y) d y
\end{gathered}
$$

Now, $\epsilon$ tends to zero, than $G_{\epsilon}$ tends to $G$ and the integral over $S_{\epsilon}$ tends to zero, thus

$$
\begin{gathered}
-T_{-\bar{a}} u+\int_{G_{\epsilon}} e^{-<\underline{a}, x-y>} K_{a_{0}}(x-y)\left(D_{y}+a\right) u(y) d y= \\
\int_{\Gamma} e^{-<\underline{a}, x-y>} K_{a_{0}}(x-y) n(y) u(y) d y .
\end{gathered}
$$

Application of $\left(D_{x}-\bar{a}\right)$ from the left leads to

$$
\begin{gathered}
\int_{\Gamma} e^{-<\underline{a}, x-y>}\left(D_{x}-a_{0}\right) K_{a_{0}}(x-y) n(y) u(y) d y+ \\
\left.\int_{G_{\epsilon}} e^{-<\underline{a}, x-y>}\left(D_{x}-a_{0}\right) K_{a_{0}}(x-y)\left(D_{y}+a\right)\right) u(y) d y= \begin{cases}u(x) & \text { in } G \\
0 & \text { in } \mathbb{R}^{m} \backslash \bar{G}\end{cases}
\end{gathered}
$$

or$$
F_{a} u+T_{a}\left(D_{x}+a\right) u=\left\{\begin{array}{ll}
u(x) & \text { in } G \\
0 & \text { in } \mathbb{R}^{m} \backslash \bar{G}
\end{array} .\right.
$$

Because the operators

$$
\begin{gathered}
D+a: W_{p, C}^{1}(G) \rightarrow L_{p, C}(G), \quad 1<p<\infty, \\
T_{a}: L_{p, C}(G) \rightarrow W_{p, C}^{1}(G), \quad 1<p<\infty
\end{gathered}
$$

are continuous, the operator

$$
F_{a}: W_{p, C}^{1-1 / p}(\Gamma) \rightarrow W_{p, C}^{1}(G)
$$

is also continuous. Thus we are able to extend the lemma and the Cauchy-Green formula:

Lemma. Let $u \in W_{p, C}^{1}(G), 1<p<\infty$, then we have

$$
\left(D_{x}+a\right) T_{a} u= \begin{cases}u(x) & \text { in } G \\ 0 & \text { in } \mathbb{R}^{m} \backslash \bar{G} .\end{cases}
$$

Theorem (Cauchy-Green formula). Let $u \in W_{p, C}^{1}(G), 1<p<\infty$, then we have

$$
F_{a} u+T_{a}\left(D_{x}+a\right) u= \begin{cases}u(x) & \text { in } G \\ 0 & \text { in } \mathbb{R}^{m} \backslash \bar{G}\end{cases}
$$

4. A note on elementary functions. If $X$ is an arbitrary element of the Cliffordalgebra $C$, then

$$
e^{X}=\sum_{n=0}^{\infty} \frac{X^{n}}{n !}=1+X+\frac{x^{2}}{2 !}+\frac{X^{3}}{3 !}+\ldots
$$


and

$$
\sinh x=\frac{e^{X}-e^{-X}}{2}=\sum_{n=0}^{\infty} \frac{X^{2 n+1}}{(2 n+1) !}, \quad \cosh X=\frac{e^{X}+e^{-X}}{2}=\sum_{n=0}^{\infty} \frac{X^{2 n}}{(2 n) !},
$$

thus

$$
e^{X}=\cosh X+\sinh X
$$

Furthermore, we have

$$
\sin X=\sum_{n=0}^{\infty}(-1)^{n} \frac{X^{2 n+1}}{(2 n+1) !}, \quad \cos X=\sum_{n=0}^{\infty}(-1)^{n} \frac{X^{2 n}}{(2 n) !} .
$$

Lemma. If $J X=X J$ for all $X$ and $J^{2}=-1$, then we have $\cosh J X=\cos X$ and $\sinh J X=J \sin X$ and $e^{J X}=\cos X+J \sin X$.

Remark. If we denote by $I_{m}=e_{1} e_{2} \ldots e_{m}$ the pseudoscalar of $C$ the only possible $J$ are the scalars $\pm i$ and the elements $\pm i I_{4 p+1}$ and $\pm I_{4 p+3}$, where $p=0,1,2, \ldots$.

If $a$ is a vector, i.e. $a=\sum_{j=1}^{m} a_{j} e_{j}$, then

$$
a^{n}=\left\{\begin{array}{ll}
|a|^{n}, & n=2 k \\
|a|^{n-1} a, & n=2 k+1
\end{array} .\right.
$$

In this case we obtain

$$
\begin{gathered}
e^{a}=\cosh a+\sinh a=\cosh |a|+\frac{a}{|a|} \sinh |a| \\
e^{J a}=e^{a J}=\cosh J a+\sinh J a=\cos a+J \sin a=\cos |a|+J \frac{a}{|a|} \sinh |a| .
\end{gathered}
$$

5. The Dirac-type operator $\frac{\partial}{\partial t} D$. We consider the equation

$$
\left(\frac{\partial}{\partial t} D\right) E=\delta(x) \otimes \delta(t)
$$

in distributional sense. We use the partial Fourier transform to compute the fundamental solution. Thus

$$
\left(\frac{\partial}{\partial t} \hat{E}+D(i y) \hat{E}\right)=l(y) \otimes \delta(t) .
$$

The solution of the problem is

$$
\hat{E}(t)=\exp (-t D(i y)) .
$$

THEOREM. The fundamental solution for the wave operator $\frac{\partial}{\partial t}+D, t \geq 0$ is

$$
E(x, t)=\left(\frac{\partial}{\partial t}-D_{x}\right) \mathcal{F}^{-1}\left(\frac{\sin |y| t}{|y|}\right),
$$

where $\mathcal{F}^{-1}\left(\frac{\sin |y| t}{|y|}\right)$ is the fundamental solution for the wave operator for $t \geq 0$.

Proof. We have seen that the partial Fourier transform of the fundamental solution is

$$
\hat{E}(t)=\exp (-t D(i y))
$$


We use the results of section 3 to get an explicit formula. We have

Thus

$$
\begin{gathered}
\exp (-t D(i y))=\exp \left(-i \sum_{j=1}^{m} e_{j} y_{j} t\right)=\cos (|y| t)-i \frac{y}{|y|} \sin (|y| t)= \\
\cos (|y| t)-\frac{D) i y)}{|y|} \sin (|Y| t)=\left(\frac{\partial}{\partial t}-D(i y)\right) \frac{\sin |y| t}{|y|} .
\end{gathered}
$$

$$
E(x, t)=\left(\frac{\partial}{\partial t}-D_{x}\right) \mathcal{F}^{-1} \frac{\sin |y| t}{|y|} .
$$

Rem ar k. The fundamental solution for the wave operator depends on the dimension $m$. In general it is a distributional derivative of a measure.

A similar problem is the following operator

$$
\frac{\partial}{\partial t}+\beta_{0}(D+a)
$$

where $\beta_{0}$ is a complex non-zero constant and $a=a_{0} e_{0}+\sum_{j=1}^{m} a_{j} e_{j}=a_{0} e_{0}+\underline{a}$.

THEOREM. The fundamental solution of

for $t \geq 0$ is

$$
\frac{\partial}{\partial t}+\beta_{0}(D+a)
$$

$$
e^{-a_{0} t<\underline{a}, x>} \cdot e^{\beta_{0}} \cdot\left(\frac{\partial}{\partial t}-D_{x}\right) \mathcal{F}^{-1}\left(\frac{\sin |y| t}{|y|}\right) .
$$

The proof is obvious.

6. The Cauchy problem for the operator $\frac{\partial}{\partial t}+D$. An important problem for this Dirac-type operator is the Cauchy problem because this problem for hyperbolic operators is well-posed.

TheOREM. The Cauchy problem for the Dirac-type operator

$$
\frac{\partial u}{\partial t}+D u=0, \quad u(x, 0)=u_{0}
$$

has a unique solution in $D^{\prime}$ for $t \geq 0$

$$
u(x, t)=\mathcal{F}^{-1}(\cos (|y| t))\langle *, x\rangle u_{0}-D_{x} \mathcal{F}^{-1}\left(\frac{\sin |y| t}{|y|}\right)\langle *, x\rangle u_{0},
$$

where $\langle *, x\rangle$ denotes the convolution only with respect to $x$. If $u_{0} \in L_{2, C}\left(\mathbb{R}^{m}\right)$ then $(u, x) \in$ $L_{2, C}\left[0, T ; \mathbb{R}^{m}\right]$.

Proof. The proof follows from the fact that $\cos (|y| t)$ and $D(i y) \frac{\sin |y| t}{|y|}$ if $t \geq 0$ are multipliers in $L_{2, C}\left(\mathbb{R}^{m}\right)$; see [DL].

To solve the inhomogeneous Cauchy problem we use again partial Fourier transform and obtain the problem

$$
\begin{gathered}
\frac{\partial \hat{u}}{\partial t}+D(i y) \hat{u}=\hat{f}, \\
\hat{u}(0)=u_{0} .
\end{gathered}
$$


Now, we set $\hat{u}=e^{-D(i y) \cdot t} v(t)$ and obtain a problem in $v$.

and the solution is

$$
e^{-D(i y) \cdot t} \frac{\partial v}{\partial t}=\hat{f}, \quad v(0)=\hat{u}(0)
$$

$$
v(t)=\hat{u}_{0}+\int_{0}^{t} e^{-D(i y) \cdot t} v(t) \hat{f}(r) d r
$$

and thus

$$
\begin{gathered}
\hat{u}(t)=e^{-D(i y) \cdot t} \hat{u}_{0}+\int_{0}^{t} e^{-D(i y)(t-r)} \hat{f}(r) d r= \\
=e^{-D(i y) \cdot t} \hat{u}_{0}+Y(t) \cdot e^{-D(i y) \cdot t}\langle *, t\rangle \hat{f}(t),
\end{gathered}
$$

where $Y(t)$ denotes the Heaviside function. To summarize we state

Theorem. The Cauchy problem

$$
\frac{\partial u}{\partial t}+D u=f(x, t), \quad u(x, 0)=u_{0}
$$

has for $u_{0} \in L_{2, C}\left(\mathbb{R}^{m}\right)$ and $f \in L_{2, C}\left[0, T ; L_{2, C}\left(\mathbb{R}^{m}\right)\right]$ a unique solution

$$
u \in L_{2, C}\left[0, T ; L_{2, C}\left(\mathbb{R}^{m}\right)\right]
$$

and

$$
\hat{u}(t)=e^{-D(i y) \cdot t} \hat{u}_{0}+Y(t) \cdot e^{-D(i y) \cdot t}\langle *, t\rangle \hat{f}(t) .
$$

7. Examples of problems with Dirac-type operators. 1) A relativistic particle with spin $1 / 2$ in an electromagnetic field with vector potential $\vec{A}$ :

$$
\sum_{k=1}^{3} e_{k}\left(i \frac{\partial}{\partial x_{t}}+b_{k}\right)+e_{0} m_{0}
$$

with rest-mass $m_{0}$ and $\left(b_{1}, b_{2}, b_{3}\right)=\vec{b}=-Q \vec{A}$, where $Q$ is the charge.

2) The Dirac "Hamilton"-operator for a free particle

$$
H=e_{0}-i \sum_{j=1} e_{j} \frac{\partial}{\partial x_{j}}
$$

and the corresponding Cauchy problem:

$$
\begin{gathered}
\frac{\partial \psi}{\partial t}+\left(\sum_{j=1}^{3} e_{j} \frac{\partial}{\partial x_{j}}+i e_{0}\right) \psi=0, \\
\psi(0)=\psi(0) .
\end{gathered}
$$

3) The equation of small perturbations for an irrotational perfect compressible gas:

$$
\begin{array}{cl}
\frac{\partial p}{\partial t}+\rho_{0} \operatorname{div} v=0, & \rho_{0} \frac{\partial v}{\partial t}+\operatorname{grad} p=0 \\
v(x, 0)=v^{0}(x), & p(x, 0)=p^{0}(x),
\end{array}
$$

is equivalent to the system

$$
\left(\frac{\partial}{\partial t}+D\right)\left(\begin{array}{c}
p^{0} \\
p_{0} v
\end{array}\right)=0
$$




$$
\left(\begin{array}{c}
p^{0} \\
p_{0} v
\end{array}\right)(x, 0)=\left(\begin{array}{c}
p^{0} \\
p_{0} v^{0}
\end{array}\right) .
$$

4) Stationary Maxwell equations:

We set $E(x, t)=E_{0}(x) e^{i \omega t} B(x, t)=B_{0}(x) e^{i \omega t}$, where $E(x, t)$ is the electrical field and $B(x, t)$ is the magnetic inductivity and then

$$
\begin{gathered}
\operatorname{div} E_{0}=\rho_{0}, \\
i \omega E_{0}-\operatorname{curl} B_{0}=-j_{0}, \\
i \omega B_{0}+\operatorname{curl} E_{0}=0, \\
\operatorname{div} B_{0}=0
\end{gathered}
$$

in the domain $G$. If we set $U=\left(0, E_{0}, B_{0}, 0\right)$, then the system above is equivalent to

$$
\left(\sum_{k=1}^{3} e_{k} \frac{\partial}{\partial x_{t}}+i \omega e_{0}\right) U=\left(\rho_{0},-j_{0} 0,0\right) .
$$

5) Time-dependent Maxwell equations in vacuum

$$
\begin{gathered}
\operatorname{div} E_{0}-\rho=0, \\
\frac{\partial E}{\partial t}-\operatorname{curl} B+j=0, \\
\frac{\partial B}{\partial t}+\operatorname{curl} E=0, \\
\operatorname{div} B=0 .
\end{gathered}
$$

If we set $V=(0, E, B, 0)$ the system above is equivalent to

$$
\left(\frac{\partial}{\partial t}+\sum_{k=1}^{3} e_{k} \frac{\partial}{\partial x_{t}}\right) V=(\rho,-j, 0,0) .
$$

\section{References}

[Be1] S. Bernstein, Analytische Untersuchungen in unbeschränkten Gebieten mit Anwendungen auf quaternionische Operatortheorie und elliptische Randwertprobleme, $\mathrm{PhD}$ Thesis, Freiberg University of Mining and Technology, 1993.

[Be2] - Cauchy-Green formulas in Clifford Analysis, to appear.

[BDS] F. Brackx, R. Delanghe and F. Sommen, Clifford Analysis, Pitman, BostonLondon-Melbourne, 1982.

[DL] R. Dautray and J.-L. Lions, Mathematical Analysis and Numerical Methods for Science and Technology, vol. 1 and 5, Springer-Verlag, 1992.

[GS] K. Gürlebeck and W. Sprößig, Quaternionic Analysis and Elliptic Boundary Value Problems, Akademie-Verlag, Berlin, 1989.

[Jan] B. Jancewicz, Multivectors and Clifford Algebra in Electrodynamics, World Scientific Publ. Co. Pt;. Ltd., 1989.

[Kr1] V. V. Kravchenko, Integral representation of biquaternionic hyperholomorphic functions and it's application PhD Thesis, Rostov State University, 1993 (Russian). 
[Kr2] -, On the generalized holomorphic vectors, Abstracts of Republican Research Conference devoted to 200 birth of N.I. Lobachevskij, Odessa, Ukraine, 1992, Part 1, p. 35.

[KS1] V. V. Kravchenko and M. V. Shapiro, Hypercomplex factorization of the multidimensional Helmholtz operator and some of its applications, Dokl. Sem. Inst. I.N. Vekua, Tbilisi 5(1) (1990), 106-109 (Russian).

[KS2] - Helmholtz operator with a quaternionic wave number and associated function theory, Deformations of Mathematical Structures. Ed. by J. Ławrynowicz, Kluwer Academic Publishers, Dordrecht 1993, 101-128.

[MP] S. G. Michlin and S. Prößdorf, Singuläre Integraloperatoren, Akademie-Verlag, Berlin, 1980.

[Ob1] E. I. Obolashvili, Space analogous of generalized analytic functions, Soobch. Akad. Nauk Gruzin. SSR, 73,1 (1974), 21-24 (Russian).

[Ob2] - Spatial generalized holomorphic vectors, Different. Uravneniya 11(1) (1975), 108115 (Russian).

[Ort] N. Ortner, Regularisierte Faltung von Distributionen. Teil 2: Eine Tabelle von Fundamentallösungen, Journal of Applied Mathematics and Physics (ZAMP), 31 (1980), 133-155.

[Xu1] Z. Xu, Boundary value problems and function-theory for Spin-invariant differential operators, PhD Thesis, State University of Gent, 1989.

[Xu2] - A function theory for the operator $D \rightarrow-\lambda$, Complex Variables Theory Appl., 16(1) (1991), 27-42. 